\title{
Evaluación de la desmineralización química de semiantracitas provenientes de minas ubicadas en Boyacá y Santander (Colombia)
}

\section{Evaluation of the chemical demineralization of semianthracites from mines located in Boyacá and Santander (Colombia)}

DOI: http://doi.org/10.17981/ingecuc.15.2.2019.05

Artículo de Investigación Científica. Fecha de Recepción: 24/09/2018. Fecha de Aceptación:04/05/2019.

\author{
Wilmer Alexander Lugo Martínez \\ Universidad del Atlántico. Barranquilla (Colombia) \\ wlugo@mail.uniatlantico.edu.co \\ Huber Yesid Avila Rios \\ Universidad del Atlántico. Barranquilla (Colombia) \\ yehuyesid@hotmail.com \\ Marley Cecilia Vanegas Chamorro \\ Universidad del Atlántico. Barranquilla (Colombia) \\ marleyvanegsas@mail.uniatlantico.edu.co \\ Alberto Albis Arrieta \\ Universidad del Atlántico. Barranquilla (Colombia) \\ albertoalbis@mail.uniatlantico.edu.co \\ Marco Antonio Ardila Barragán \\ Universidad Pedagógica y Tecnológica de Colombia. Tunja (Colombia) \\ marco.ardila@uptc.edu.co
}

Para citar este artículo:

Wilmer Alexander Lugo-Martínez; Huber Yesid Avila-Rios; Marley Cecilia Vanegas-Chamorro; Alberto Albis-Arrieta; Marco Antonio ArdilaBarragán. "Evaluación de la desmineralización química de semiantracitas provenientes de minas ubicadas en Boyacá y Santander (Colombia)" INGE CUC, vol. 15, no. 2, pp. 47-55, 2019. DOI: http://doi.org/10.17981/ingecuc.15.2.2019.05

\section{Resumen}

Introducción- El uso no energético de carbones de alto rango (antracitas) tiene un gran potencial en industrias tales como la metalurgia y en la síntesis de nuevos materiales carbonosos. Sin embargo, antes de su uso en estas aplicaciones, estos deben ser tratados para eliminar impurezas o compuestos no deseados.

Objetivo- Evaluar la eficiencia del proceso de desmineralización química de semiantracitas mediante el uso de diferentes ácidos variando las condiciones de operación del proceso.

Metodología- Se realizó la caracterización química de dos muestras: Boavita (B) y Capitanejo (C) provenientes de minas de Boyacá y Santander (Colombia), respectivamente. Se evaluó la remoción de cenizas y materia mineral de las muestras utilizando $[\mathrm{HCl}]=5 \mathrm{M}, \mathrm{HF} 40 \%$ y $\mathrm{HCl} 38 \%$ a dos diferentes tiempos de reacción (45 y 60 minutos) y dos tamaños de partícula del material (250 y $500 \mu \mathrm{m})$.

Resultados- Los valores mínimos de contenido de cenizas (bs) alcanzados mediante el proceso de desmineralización para las muestras $\mathrm{B}$ y $\mathrm{C}$, fueron 0,65 y $0,76 \%$ respectivamente, los cuales se obtuvieron con tamaño de partícula de $250 \mu \mathrm{m}$ y 60 minutos de exposición en cada uno de los ácidos empleados en este estudio.

Conclusiones- A menor tamaño de partícula se incrementa la superficie de contacto y mejora el grado de desmineralización, independientemente del tiempo de exposición a los ácidos. La eficiencia del beneficio químico muestra rendimientos en la reducción de silicatos, aluminatos y aluminosilicatos al 100\%, mientras que para minerales férricos está por encima del 50\%.

Palabras clave- Antracitas; beneficio químico; materia mineral; desmineralización; ácido clorhídrico; ácido fluorhídrico.

\section{Abstract}

Introduction- The non-energy use of high-range carbons (an thracite) has great potential in industries such as metallurgy and in the synthesis of new carbonaceous materials. However, before being used in these applications, they must be treated to remove impurities or unwanted compounds.

Objective- To evaluate the efficiency of the process of chemical demineralization of semianthracites through the use of different acids varying the operating conditions of the process.

Method-Two samples were chemically characterized: Boavita (B) and Capitanejo (C) from the Boyacá and Santander (Colombia) mines, respectively. Ash and mineral matter removal from the samples was evaluated using $[\mathrm{HCl}]=5 \mathrm{M}, \mathrm{HF} 40 \%$ and $\mathrm{HCl}$ $38 \%$ at two different reaction times (45 and 60 minutes) and two particle sizes of the material (250 and $500 \mu \mathrm{m})$.

Results - The minimum values of ash content (bs) reached through the demineralization process for samples $\mathrm{B}$ and $\mathrm{C}$, were 0.65 and $0.76 \%$ respectively, which were obtained with a particle size of $250 \mu \mathrm{m}$ and 60 minutes of exposure in each of the acids used in this study.

Conclusions- A smaller particle size increases the contact surface and improves the degree of demineralization, regardless of the time of exposure to acids. The efficiency of the chemical benefit shows yields in the reduction of silicates, aluminates and aluminosilicates to $100 \%$, while for ferric minerals it is above $50 \%$.

Keywords- Anthracite; chemical beneficiat; mineral matter; demineralization; Hydrochloric acid; Hydrofluoric acid. 


\section{INTRODUCCIÓN}

La antracita es el carbón mineral de más alto rango por su alto contenido de carbono fijo y menor contenido de humedad, ceniza y materia volátil. Se subdivide en tres categorías a saber: meta-antracitas, antracitas y semiantracitas, siendo esta última la de menor contenido de carbono. Tienen por lo general iguales o superiores a $86 \%$ en masa de carbono, el cual se encuentra distribuido en una estructura macromolecular de anillos aromáticos condensados, formando grandes unidades enlazadas por grupos aromáticos principalmente, confiriéndole un cierto grado de orden estructural [1]. Por el alto contenido de carbono y baja proporción de cadenas alifáticas, se puede considerar como una excelente alternativa para la producción de materiales de carbono con mayor valor agregado.

Infortunadamente, los yacimientos de carbón poseen constituyentes inorgánicos e impurezas que pueden afectar o perjudicar el comportamiento de ese combustible fósil durante los procesos de transformación a los que se expone para poder aprovechar a nivel industrial, razón por la cual se deben beneficiar. Los procesos de beneficio del carbón se realizan para reducir contenidos de materia mineral e inerte de su estructura, y por tanto mejorar sus propiedades fisicoquímicas. Las tecnologías de beneficio incluyen las tecnologías físicas, químicas, fisicoquímicas y de bio-beneficio. Los métodos físicos son separación por gravedad y separación magnética. Los métodos químicos son lixiviación química por ácido o álcali, mientras que los métodos fisicoquímicos son la flotación y la aglomeración. Las tecnologías de biobeneficio se logran mediante el uso de bacterias específicas. A veces la combinación de dos o tres tecnologías de beneficio son necesarias para lograr una limpieza efectiva de carbones [2]. El beneficio físico del carbón se basa en las diferencias de las propiedades físicas de la materia orgánica con respecto a la materia mineral. Como ejemplos se mencionan la molienda, el tamizado, el uso de ciclones e hidrociclones, separación en medios densos, gravimetría, flotación, entre otras. Las ventajas y limitaciones de estas técnicas se definen de acuerdo a la propiedad física que se manipula o de la aplicación que se requiera [3].

Por otra parte, los procedimientos de beneficio químico se aplican cuando se requiere solubilizar constituyentes minerales presentes en el carbón o para transformarlos a formas solubles para facilitar su extracción [4]. La diferencia más notable entre los procesos de tratamiento, es que mediante el procedimiento químico ocurren cambios en la estructura del carbón debido a la acción de agentes desmineralizantes, mientras con el físico no; adicionalmente, la limpieza física resulta eficiente en la remoción de materia mineral y parte del azufre inorgánico; por esta razón, la mayoría de técnicas de beneficio químico se emplean principalmente para la reducción de azufre y otros elementos presentes en mayor proporción, tales como; $\mathrm{Si}, \mathrm{Na}, \mathrm{K}, \mathrm{Al}, \mathrm{Fe}$, Ca y Mg que se encuentran enlazados o interactuando químicamente con la estructura del carbón [3], [5], [6].

Debido a la baja desmineralización lograda a través del uso de técnicas físicas de beneficio para eliminar del carbón la mayoría de las impurezas, los procesos químicos son frecuentemente empleados. El esquema general seguido para limpiar el carbón involucra la lixiviación, teniendo en cuenta una variedad de condiciones que dependen de la composición del carbón que será tratado. El empleo de soluciones ácidas y alcalinas ha demostrado ser eficaz para reducir cantidades significativas de materia mineral. Los procesos de desmineralización, ya sean aplicados de forma independiente o después de los procesos de limpieza física, han sido ampliamente explorados para la producción de carbón limpio y ultra limpio [7].

La revisión exhaustiva de la literatura científica, muestra como la desmineralización del carbón puede mejorarse mediante los procesos de desmineralización química. Varios autores han estudiado la desmineralización de carbones con bajo contenido de materia mineral para obtener materiales carbonosos ultra limpios [3] [4] [6] [8] [17]. En esta investigación, se empleó un beneficio químico con $\mathrm{HCl} 5 \mathrm{M}$, $\mathrm{HF} 40 \%$ y $\mathrm{HCl} 38 \%$ a dos diferentes tiempos de reacción (45 y 60 minutos) y dos tamaños de partícula (250 y $500 \mu \mathrm{m}$ ) de dos semi-antracitas con el fin de evaluar la eficiencia del proceso desmineralización química mediante el uso de estos ácidos variando las condiciones de operación del proceso de cara a la obtención de materiales con mayor valor agregado.

\section{Metodología}

\section{A. Origen de las muestras}

Para realizar este trabajo se utilizaron dos muestras de semi-antracitas colombianas denominadas Boavita (B) y Capitanejo (C); provienen de las minas El Sauzal ubicada a $18 \mathrm{~km}$ del municipio de Boavita (Boyacá, Colombia) y San Luis en jurisdicción del municipio El Carmen de Chucurí (Santander, Colombia). Se seleccionó este tipo de carbón por el alto contenido de carbono fijo, bajo contenido de materia volátil y propiedades no aglomerantes, ideal para la obtención de nuevos materiales carbonosos.

\section{B. Preparación de las muestras}

La muestra $B$ se recibió con tamaño de partícula entre 0 y $2 \mathrm{~mm}$, y la muestra $\mathrm{C}$ entre 2 y $6 \mathrm{~mm}$. La preparación mecánica de los carbones se realizó a partir de muestras representativas, para caracterización bajo normas ASTM, y para el proceso de beneficio químico reduciéndolas a tamaños entre 500 y $200 \mu \mathrm{m}$, y por debajo de $250 \mu \mathrm{m}$. 


\section{Caracterización de las muestras}

La caracterización se llevó a cabo mediante análisis próximo para determinar humedad, cenizas, materia volátil y carbono fijo (normas ASTM D3172 a D3175); análisis último para determinar $\mathrm{C}, \mathrm{H}, \mathrm{N}$ y $\mathrm{O}$ (norma ASTM D3176); determinación de azufre total (norma ASTM D4239); y determinación de poder calorífico (norma ASTM D5865); también se realizó la caracterización de la materia orgánica mediante la técnica de Espectroscopía Infrarroja con Transformada de Fourier (FTIR) por tramitancia y de la materia inorgánica utilizando la técnica de Fluorescencia de Rayos X (XRF) directamente en las muestras de carbón.

\section{Beneficio químico (Tratamiento de desmineralización)}

Las semi-antracitas se sometieron a tratamientos de desmineralización química utilizando ácido clorhídrico y ácido fluorhídrico. Este tratamiento de desmineralización profunda se realizó según la Norma ISO 602 (temperatura de reacción de $60{ }^{\circ} \mathrm{C} \pm 3^{\circ} \mathrm{C}$, y relación carbón (g) - agente desmineralizante de $1: 4$ para $\mathrm{HCl} 5 \mathrm{M}$ y $\mathrm{HF}$ 40\% y 1:5 para $\mathrm{HCl} 38 \%$ ). Posteriormente los residuos se lavaron con agua hasta alcanzar un $\mathrm{pH}$ mayor a 4.5. El mezclado y el control de la temperatura se realizaron en un ultrasonido Branson M2800 CPX-HE.

Para determinar las mejores condiciones de desmineralización se consideraron dos variables de estudio: tiempo de reacción y tamaño de partícula. Los valores de tiempo empleado fueron de 45 y 60 minutos, mientras que el tamaño de partículas fue de 250 y $500 \mu \mathrm{m}$. Usando estos factores y niveles se realizó un diseño de experimento factorial $2^{2}$ con bloques aleatorios con el porcentaje final de cenizas en el carbón como respuesta.

Los resultados del beneficio químico se analizaron utilizando la correlación de Parr, siguiendo la metodología planteada en la norma ASTM D388-18a. La correlación de Parr (1) se usa para estimar la materia mineral presente en carbones mediante el porcentaje de cenizas y el porcentaje de azufre total presente en las muestras de partida y desmineralizadas [9].

$$
\mathrm{MM}=1.08 \mathrm{~A}+0.55 \mathrm{~S}
$$

En (1), A y S corresponden a los porcentajes de cenizas y azufre total determinados por las normas ASTM D3174 y D4239, respectivamente. Esta correlación supone que los minerales de arcilla, con un contenido medio de agua aguade hidratación del 8\%, y la pirita, que contiene esencialmente todo el azufre, son los únicos grupos minerales que se consideran presente en las muestras de forma apreciable. También, para esta correlación se suponen las siguientes reacciones durante la combustión: los grupos hidroxilo de los minerales arcillosos se desprenden a la atmósfera, el azufre se convierte en dióxido de azufre volátil y la pirita se descompone en óxido de hierro el cual se retiene en las cenizas [9]. La correlación de Parr intenta corregir la ceniza y el azufre medidos para estas reacciones ajustando su masa a la de los minerales originales en la muestra estudiada.

\section{Resultados y Discusión}

\section{A. Caracterización general}

En la Tabla 1 se presentan los resultados de los análisis de las semi-antracitas de partida B y C, realizados siguiendo las normas ASTM. De acuerdo con los resultados obtenidos, se puede apreciar como las semi-antracitas estudiadas son bastante similares. En el análisis elemental, la diferencia no supera el $0.5 \%$ de masa entre ambas muestras, aunque estas tengan sus orígenes en yacimientos diferentes. El contenido de azufre se encuentra entre el 1 y $2 \%$. Cabe destacar el bajo contenido de humedad, cenizas y de material volátil de las muestras, lo que puede ser atribuido al hecho de ser carbones de alto rango.

Tabla 1. Caracterización general en base Seca DE LAS MUESTRAS ESTUDIADAS.

\begin{tabular}{|c|c|c|}
\hline Análisis/Parámetro & Boavita & Capitanejo \\
\hline \multicolumn{3}{|c|}{ Análisis próximo (\%m) } \\
\hline Humedad & 1.92 & 1.17 \\
\hline Cenizas & 9.03 & 10.62 \\
\hline Materia Volátil & 13.11 & 11.75 \\
\hline \multicolumn{2}{|c|}{ Análisis Elemental (\%m) } \\
\hline Carbono & 81.06 & 80.94 \\
\hline Hidrógeno & 3.41 & 3.33 \\
\hline Nitrógeno & 1.53 & 1.48 \\
\hline Oxígeno & 14.00 & 14.25 \\
\hline Azufre & 1.72 & 1.36 \\
\hline \multicolumn{2}{|c|}{ Poder Calorífico (BTU) } \\
\hline Poder Calorífico & 13779 & 13691 \\
\hline
\end{tabular}

Fuente: Autores.

Teniendo en cuenta el análisis elemental realizado a las muestras, se puede decir que el contenido en carbono es levemente menor al esperado por ser una semiantracita [1], también se puede destacar los porcentajes en masa de los elementos más combustibles de las muestras ( $\mathrm{C}$ y H), dando como resultado $81.06 \%$ y $3.41 \%$ respectivamente para Boavita, mientras que para Capitanejo los valores fueron $80.94 \%$ y $3.33 \%$.

\section{B. Caracterización de minerales}

En la Tabla 2 se muestran los resultados de los análisis de materia mineral de las dos semi-antracitas estudiadas. La semi-antracita $\mathrm{C}$ posee un contenido de silicio un poco mayor, siendo habitual en carbones de este tipo que este sea el elemento inorgánico presente en mayor proporción en ambas muestras, seguido del aluminio y el hierro. La relación Si/Al es cercana en ambas semi- 


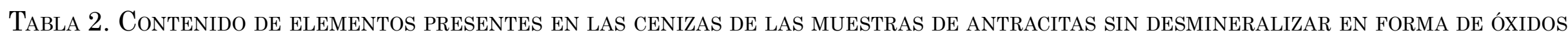
(PORCENTAJE).

\begin{tabular}{|c|c|c|c|c|c|c|c|c|c|c|c|c|c|}
\hline & $\mathrm{SO}_{3}$ & $\mathrm{SiO}_{2}$ & $\mathrm{Al}_{2} \mathrm{O}_{3}$ & $\mathrm{TiO}_{2}$ & $\mathrm{Fe}_{2} \mathrm{O}_{3}$ & $\mathrm{CaO}$ & $\mathrm{MgO}$ & $\mathrm{K}_{2} \mathrm{O}$ & $\mathrm{Na}_{2} \mathrm{O}$ & $\mathrm{P}_{2} \mathrm{O}_{5}$ & $\mathrm{SrO}$ & $\mathrm{BaO}$ & $\mathrm{MnO}_{2}$ \\
\hline $\mathrm{B}$ & 0.96 & 54.980 & 21.426 & 1.01 & 17.786 & 0.940 & 0.534 & 1.107 & 0.417 & 0.401 & 0.063 & 0.050 & 0.032 \\
\hline $\mathrm{C}$ & 0.54 & 58.96 & 21.11 & 1.13 & 12.07 & 0.52 & 1.87 & 0.65 & 0.24 & 0.21 & 0.04 & 0.04 & 0.03 \\
\hline
\end{tabular}

Fuente: Autores.

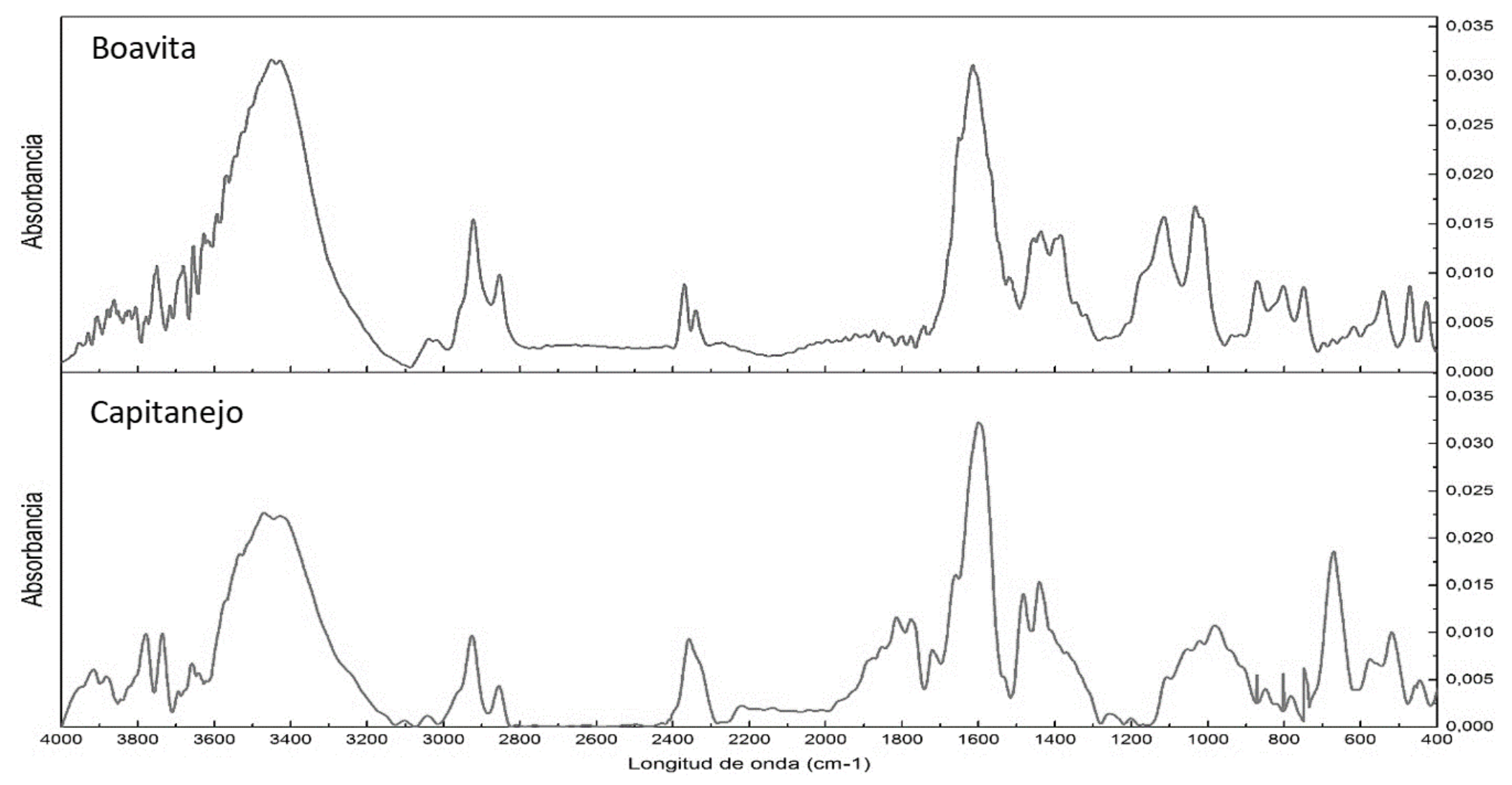

Fig. 1. Espectros infrarrojos para las muestras B y C de antracitas.

Fuente: Autores.

antracitas sugiriendo la presencia de proporciones similares de silicio combinado en forma de arcillas y no combinado en forma de cuarzo para ambas. Se puede observar también que las concentraciones de óxidos de $\mathrm{Ca}, \mathrm{K}, \mathrm{P}, \mathrm{Sr}, \mathrm{Na}$ y S son aproximadamente el doble en $\mathrm{B}$; la concentración del óxido de magnesio en $\mathrm{C}$ es aproximadamente 3.5 veces la concentración de B. Los contenidos de Ba y Mn se pueden considerar comparables en ambas muestras.

C. Caracterización de las muestras de partida por espectroscopía infrarroja con transformada de Fourier (FTIR)

En la Fig. 1 se presentan los espectros infrarrojos de las semi-antracitas de partida B y C. Los espectros de ambas muestras presentan bandas poco intensas en el intervalo de número de ondas comprendido entre 3694 y $3625 \mathrm{~cm}^{-1}$, las cuales se encuentran asociadas con vibraciones de tensión del enlace $\mathrm{O}-\mathrm{H}$ unido a especies de aluminio en arcillas. También se observa la banda característica de la vibración de tensión del grupo SiO-H o enlaces silanol [10]. Los grupos silanol contribuyen a las bandas de vibraciones de tensión de enlaces $\mathrm{O}-\mathrm{H}$, las cuales pueden aparecer entre 3700 y $3200 \mathrm{~cm}^{-1}$.
En los espectros aparecen dos picos muy intensos a 3450 y $3428 \mathrm{~cm}^{-1}$ para B y 3472 y $3430 \mathrm{~cm}^{-1}$ para $\mathrm{C}$, correspondientes a las vibraciones de tensión de los enlaces de hidrógeno de los hidroxilos presentes en forma de grupos carboxilos, fenol o alcohol [11]. La presencia de estas bandas en el espectro indica que en la muestra hay grupos hidroxilo o combinaciones de estos, así como agua presente en la muestra como humedad intrínseca o absorbida durante la preparación de la pastilla con $\mathrm{KBr}[12]$.

Se puede observar un pico de baja intensidad en aproximadamente $3030 \mathrm{~cm}^{-1}$ en ambas muestras, el cual es característico de vibraciones de tensión $\mathrm{C}-\mathrm{H}$ en compuestos aromáticos. En esta zona también se pueden encontrar bandas correspondientes a vibraciones de tensión $\mathrm{C}-\mathrm{H}$ de alquenos, al igual que las vibraciones de tensión $\mathrm{O}-\mathrm{H}$ y N-H. Sin embargo, las bandas que deben presentarse para esos dos últimos enlaces deben ser mucho más anchos, por lo que se podría intuir que esta banda corresponde a las vibraciones de tensión $\mathrm{C}-\mathrm{H}$ en aromáticos. El grupo metilo $\left(\mathrm{CH}_{3}\right)$ provoca dos vibraciones de tensión $\mathrm{C}-\mathrm{H}$, una simétrica y otra asimétrica en las longitudes de onda próximas a 2850 y $2960 \mathrm{~cm}^{-1}$ respectivamente. El grupo metileno $\left(\mathrm{CH}_{2}\right)$ también posee vibraciones simétrica y asimétrica. Estas vibraciones 
se encuentran aproximadamente en las longitudes de ondas 2922 y $2855 \mathrm{~cm}^{-1}$ respectivamente [Referencia]. En los espectros se puede apreciar que ambas muestras presentan las cuatro vibraciones de tensión siendo en $\mathrm{B}$ más intensa que en $\mathrm{C}$.

La zona del espectro comprendida entre los números de onda 2000 a $1700 \mathrm{~cm}^{-1}$ es característica del grupo funcional carbonilo presente en compuestos tales como aldehídos $(-\mathrm{CHO})$, cetonas $(\mathrm{C}=\mathrm{O})$, ácidos carboxílicos $(-\mathrm{COOH})$, ésteres $(-\mathrm{CO}-\mathrm{O}-)$, aminas $(-\mathrm{CO}-\mathrm{N})$, anhídridos (-CO-C-CO-), etc. [10]. Se puede apreciar en la Fig. 1 que en el espectro de $\mathrm{C}$ se encuentran bandas características que no se observan en el espectro de B, esto sugiere que $\mathrm{C}$ posee más grupos funcionales del tipo carbonilo y sus conjugaciones, que no se encuentran presentes en B. Se pueden observar bandas de baja intensidad en el intervalo $1800-1700 \mathrm{~cm}^{-1}$ en B y bandas de alta intensidad en $\mathrm{C}$ características de las lactonas debido a la tensión de sus grupos $\mathrm{C}=\mathrm{O}$ y $\mathrm{C}-\mathrm{O}$. La banda característica para las vibraciones de tensión de la lactona se presenta entre las longitudes de onda 1840-1815 $\mathrm{cm}^{-1}$, podría decirse que en la muestra C hay presencia de estos grupos, lo cual no sucede con B ya que las bandas presentes son muy débiles y pueden ser por presencia de estas especies o ruido externo que afecta la medición [13].

La banda más definida en los espectros infrarrojos es la ubicada a $1600 \mathrm{~cm}^{-1}$ y se atribuye principalmente a la vibración de tensión de los enlaces $\mathrm{C}=\mathrm{C}$ en anillos aromáticos. Cuando el anillo es sustituido por grupos alquil, en la banda a $1600 \mathrm{~cm}^{-1}$ aparece un hombro a aproximadamente $1580 \mathrm{~cm}^{-1}$. En los espectros de ambas muestras no se logran apreciar estos hombros [14].

Las bandas comprendidas entre los números de onda $1470-1365 \mathrm{~cm}^{-1}$ corresponden a las vibraciones de tensión de los enlaces $\mathrm{C}-\mathrm{H}$ de los compuestos alifáticos. Ambas muestras presentan una sola banda en común en esta región la cual se encuentra aproximadamente a $1434 \mathrm{~cm}^{-1}$ y podría atribuirse tanto a vibraciones de deformación de enlaces $\mathrm{C}-\mathrm{H}$ en alifáticos, principalmente alcanos como a la presencia de aniones $\mathrm{CO}_{2}$ - presentes en compuestos como la calcita. La muestra $\mathrm{B}$, presenta una banda a $1382 \mathrm{~cm}^{-1}$ la cual posiblemente corresponda a la vibración de tensión simétrica $\mathrm{C}-\mathrm{H}$ del grupo metilo $\left(-\mathrm{CH}_{3}\right)$ en cadenas alifáticas. La muestra $\mathrm{C}$ presenta una banda definida a $1480 \mathrm{~cm}^{-1}$ y puede asociarse con vibraciones de tensión asimétrica $\mathrm{C}-\mathrm{H}$ del grupo metilo $\left(-\mathrm{CH}_{3}\right)$ en cadenas alifáticas [10].

En la última zona del espectro (1300-400 $\left.\mathrm{cm}^{-1}\right) \mathrm{de}$ ambas muestras, se encuentra una superposición de bandas de componentes orgánicos e inorgánicos lo cual resulta ser negativo para esta técnica considerando que se busca caracterizar la materia orgánica presente en las muestras. En esta zona se encuentran bandas en las dos muestras, las cuales pueden corresponder a vibraciones de tensión del enlace $\mathrm{C}-\mathrm{O}$ en alcoholes, vibraciones de tensión del enlace asimétrico $\mathrm{C}-\mathrm{O}-\mathrm{C}$ en éteres y vibraciones de tensión simétricas $\mathrm{C}-\mathrm{O}-\mathrm{C}$ en ésteres o vibraciones de tensión del enlace $\mathrm{Si}-\mathrm{O}$ o vibraciones de tensión asimétrica de la cadena $\mathrm{Si}-\mathrm{O}-\mathrm{Si}$ en minerales como cuarzo y silicatos tales como la Kaolinita e Illita [13].

En el intervalo $900-700 \mathrm{~cm}^{-1}$ hay tres bandas definidas para $\mathrm{B}$ y $\mathrm{C}$ las cuales se encuentran aproximadamente a 869,803 y $746 \mathrm{~cm}^{-1}$ las cuales se atribuyen a las vibraciones de deformaciones fuera del plano de los enlaces $\mathrm{C}-\mathrm{H}$ en compuestos aromáticos. En la última zona del espectro comprendida entre 650 y $400 \mathrm{~cm}^{-1}$, se presentan bandas que se atribuyen a vibraciones de constituyentes de la materia mineral de las muestras tales como arcillas y cuarzo.

\section{Beneficio químico (Tratamiento de desmineralización)}

La nomenclatura de las muestras estudiadas se consideró de la siguiente manera: todas las muestras se indentifican con la letra "C" seguida de tres números indicativos siendo el primer subíndice es 0 para Boavita y 1 para Capitanejo, el subíndice 2 es 0 para el menor tamaño de partícula $250 \mu \mathrm{m}$ y 1 para el mayor $500 \mu \mathrm{m}$. El tercer subíndice es 0 para el menor tiempo 45 min y es 1 para el mayor tiempo $60 \mathrm{~min}$.

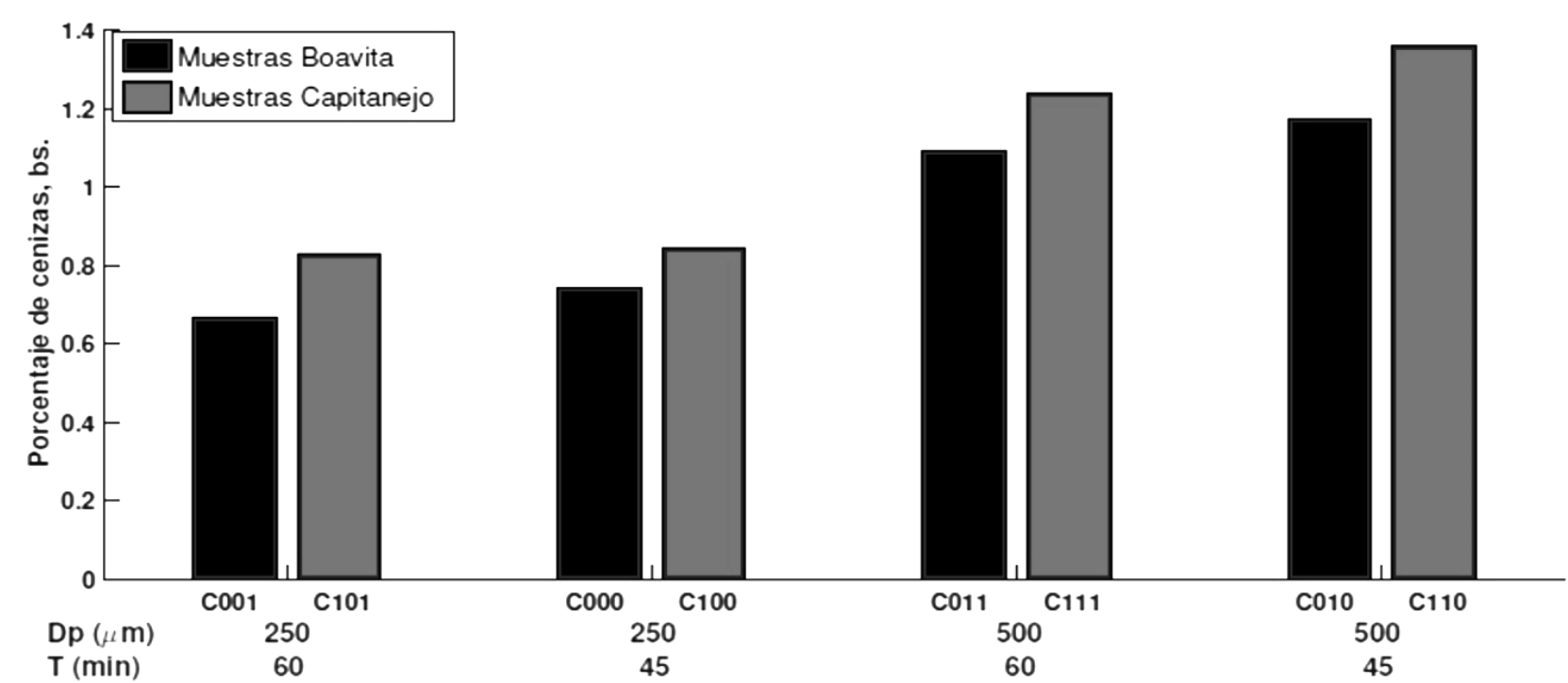

Fig. 2. Porcentaje de cenizas para las muestras de carbones desmineralizados.

Fuente: Autores. 
La Fig. 2 ilustra el comportamiento que tiene el porcentaje de cenizas en las muestras cuando se modifican las variables de entrada (tamaño de partícula y tiempo) en los tratamientos químicos. Se puede apreciar que existe una tendencia similar para las cenizas sin importar la procedencia de la misma. Al disminuir el tamaño de partícula también disminuye el porcentaje de cenizas presente en las muestras desmineralizadas, destacándose que las muestras con tamaños de partículas de 500 $\mu \mathrm{m}$ poseen un porcentaje de cenizas por encima del $1 \%$ mientras que las de tamaño de partícula de $250 \mu \mathrm{m}$ poseen un porcentaje de cenizas por debajo de $0.9 \%$.

El efecto del tiempo no es muy apreciable para las muestras tratadas, viéndose una diferencia en las cenizas para B tan solo de $0.07 \%$ y de $0.08 \%$ para las muestras de 250 y $500 \mu \mathrm{m}$ respectivamente, para los tiempos de 60 y 45 minutos. Para el caso de las muestras de C, se obtuvo una diferencia del $0.12 \%$ para las cenizas de las muestras de $500 \mu \mathrm{m}$ a 60 y 45 minutos, sin embargo, es tan solo del $0.016 \%$ para las muestras con un tamaño de partícula de $250 \mu \mathrm{m}$. Esto indica que el efecto del tiempo no es relevante cuando el tamaño de partícula disminuye en las muestras provenientes de C.

Con el software estadístico STATGRAPHICS Centurión XVI.I se encontró un modelo matemático que correlaciona la variable dependiente o de respuesta $(\mathrm{Y})$ con las variables independientes o de entrada $\left(\mathrm{X}_{1}, \mathrm{X}_{2}\right)$ de la forma como se muestra en la ecuación 2 . Los coeficientes b para las muestras de B y C se encuentran tabulados en la Tabla 3.

$$
(1 / Y)=b_{0}+b_{1} X_{1}+b_{2} X_{2}+b_{12} X_{1} X_{2}
$$

TABla 3. CoEficientes DEL MODELO DE REGREsióN PARA EL BENEFICIO QUÍMICO.

\begin{tabular}{|c|c|c|}
\hline Coeficientes & B & C \\
\hline $\mathrm{b}_{0}$ & 1,15941 & 0,994414 \\
\hline $\mathrm{b}_{1}$ & $-0,26138$ & $-0,209146$ \\
\hline $\mathrm{b}_{2}$ & 0,0454672 & 0,0190012 \\
\hline $\mathrm{b}_{12}$ & $-0,0243415$ & 0,00569583 \\
\hline
\end{tabular}

Fuente: Autores.

En la Tabla 4 y Tabla 5 se presenta un resumen de los resultados del ANOVA realizado a los modelos obtenidos. Aquí, las magnitudes de los coeficientes del modelo indican la contribución de cada término en el valor de la variable de salida; esto se corrobora teniendo en cuenta la significancia de cada término respecto a cada valor de la razón $\mathrm{F}$.

A manera de ejemplo, se observa que para las muestras de Boavita (Tabla 4), el valor de la razón F correspondiente a la variable A (tamaño de partícula) excede 33 veces la magnitud de la razón $\mathrm{F}$ de la variable B (tiempo), indicando que la variable A es la más influyente en la eficiencia del método de remoción de la materia mineral. Este comportamiento también se evidencia para las muestras de Capitanejo al observar la Tabla 5, donde el valor de F para la variable A es 46 veces mayor que el valor $F$ para la variable $B$. De la misma forma, los coeficientes de la Tabla 3 muestran que $b_{1}$ es mayor que los coeficientes $b_{2} \mathrm{y} \mathrm{b}_{12}$, corroborando lo anteriormente expuesto.

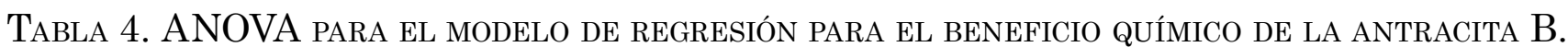

\begin{tabular}{|c|c|c|c|c|c|}
\hline Fuente & Suma de Cuadrados & Gl & Cuadrado Medio & Razón-F & Valor P \\
\hline A: Diámetro de partícula & 0,546558 & 1 & 0,546558 & 39,57 & 0,0033 \\
\hline B: Tiempo & 0,0165381 & 1 & 0,0165381 & 1,20 & 0,3353 \\
\hline AB & 0,00474007 & 1 & 0,00474007 & 0,34 & 0,5894 \\
\hline Error total & 0,0552432 & 4 & 0,0138108 & & \\
\hline Total (corr.) & 0,623079 & 7 & & & \\
\hline
\end{tabular}

Fuente: Autores.

TABla 5. ANOVA PARA El MODELO DE REGREsión PARA El BENEFICIO QUímico DE LA ANTRACITA C.

\begin{tabular}{|c|c|c|c|c|c|}
\hline Fuente & Suma de Cuadrados & Gl & Cuadrado Medio & Razón-F & Valor P \\
\hline A: Diámetro de partícula & 0,430917 & 1 & 0,430917 & 11,99 & 0,0258 \\
\hline B: Tiempo & 0,00940506 & 1 & 0,00940506 & 0,26 & 0,6359 \\
\hline AB & 0,00552826 & 1 & 0,00552826 & 0,15 & 0,7149 \\
\hline Error total & 0,14378 & 4 & 0,035945 & & \\
\hline Total (corr.) & 0,58963 & 7 & & & \\
\hline
\end{tabular}

Fuente: Autores. 

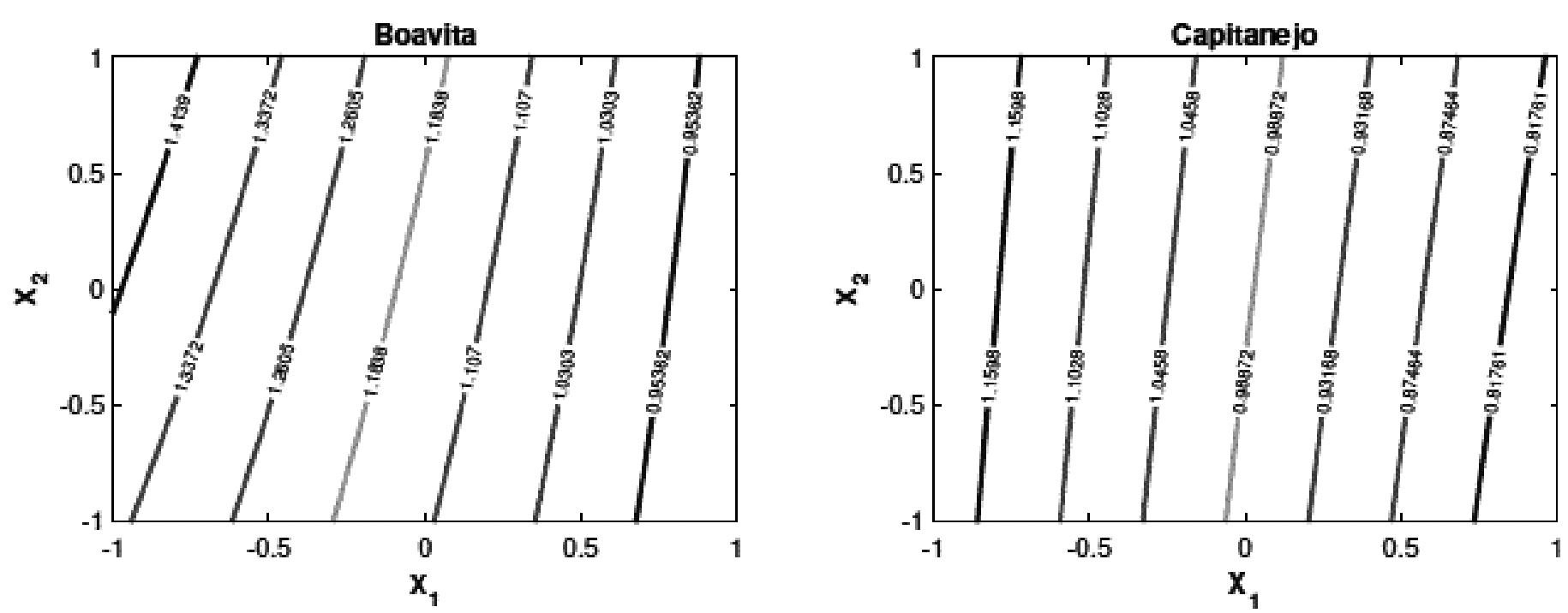

Fig. 3. Curvas de nivel para el modelo de regresión del beneficio químico de las antracitas.

Fuente: Autores

Esta tendencia se hace evidente al observar la Fig. 2 , donde se puede apreciar que las diferencias de los valores de cenizas son mayores entre tamaños de partículas en la misma muestra en comparación con las diferencias al tener en cuenta solo el tiempo.

El modelo encontrado que se ajusta mejor a los datos experimentales obtenidos fue el inverso del porcentaje de ceniza (1/Y). Teniendo esto en cuenta, para encontrar las mejores condiciones de desmineralización se estableció como objetivo maximizar dicha variable, lo que a su vez significa minimizar el porcentaje de ce$\operatorname{nizas}(\mathrm{Y})$.

También se determinaron las curvas de nivel del modelo ajustado para cada muestra y a su vez se determinaron las mejores condiciones de operación en las cuales se logra el objetivo de minimizar las cenizas de las muestras. En la Fig. 3 se presentan las curvas de nivel obtenidas para las muestras de B y C. Estas son una representación en un plano de líneas constantes de la variable de respuesta $(1 / \mathrm{Y})$ en función de las variables de entrada estandarizadas $\left(\mathrm{X}_{1} \mathrm{y}_{2}\right)$. Adicionalmente se observan los valores correspondientes a cada línea, los cuales representan el inverso de las cenizas $(1 / \mathrm{Y})$.

Se pudo determinar que los mejores resultados para las condiciones estudiadas corresponden a un tamaño de partícula de $250 \mu \mathrm{m}$ y 60 minutos, siendo este punto el de mayor valor para $1 / \mathrm{Y}$ y por tanto el menor valor para las cenizas. Para ambas muestras el resultado es el mismo.

\section{E. Determinación de elementos presentes en las muestras beneficiadas quimicamente}

La Tabla 6 y la Tabla 7 muestran la concentración de los elementos presentes en las muestras de B y C, respectivamente.

Del análisis se destaca la reducción del 100\% de los elementos aluminio y silicio en todas las muestras beneficiadas a excepción de la muestra C000 cuya re- ducción fue del 10\% aproximadamente. La presencia de cloro en las muestras beneficiadas se debe a la inclusión del cloro a la estructura del carbón, así como la formación de enlaces con los minerales presentes en la muestra, el cual proviene del lavado con ácido clorhídrico [3].

Analizando las muestras beneficiadas a las mejores condiciones de desmineralización, se puede apreciar que el contenido más bajo de hierro también se consigue en este punto, obteniéndose una reducción del $52.69 \%$ y $68.75 \%$ para B y C, respectivamente. Este comportamiento se mantiene para el potasio, calcio, cromo, manganeso, selenio y estroncio para B y calcio, titanio, manganeso, cobre, bromo y estroncio para $\mathrm{C}$. Las concentraciones de galio y molibdeno presentes en los análisis no se tuvieron en cuenta ya que el galio es el elemento utilizado como estándar interno y la fuente de rayos $\mathrm{X}$ contiene molibdeno, interfieriendo con su cuantificación.

TAbla 6. Concentración de minerales PResentes en muestras DE PARTIDA Y BENEFICIADAS QUímICAMENTE PROVENIENTE DE B.

\begin{tabular}{|c|c|c|c|c|c|}
\hline Elemento & Partida & $\mathrm{C} 000$ & $\mathrm{C} 001$ & $\mathrm{C} 010$ & $\mathrm{C} 011$ \\
\hline $\mathrm{Al}$ & 7.533 & 6.8 & 0 & 0 & 0 \\
\hline $\mathrm{Si}$ & 16.333 & 0 & 0 & 0 & 0 \\
\hline $\mathrm{Cl}$ & 0 & 20.35 & 12.43 & 10.985 & 15.85 \\
\hline $\mathrm{K}$ & 0.649 & 0 & 0 & 0.015 & 0.808 \\
\hline $\mathrm{Ca}$ & 0.476 & 0.542 & 0.091 & 0.096 & 0.336 \\
\hline $\mathrm{Ti}$ & 0.670 & 0.665 & 0.425 & 0.335 & 0.439 \\
\hline $\mathrm{Cr}$ & 0.004 & 0.038 & 0 & 0 & 0.012 \\
\hline $\mathrm{Mn}$ & 0.014 & 0.102 & 0 & 0 & 0 \\
\hline $\mathrm{Fe}$ & 10.453 & 5.570 & 4.945 & 6.360 & 7.44 \\
\hline $\mathrm{Cu}$ & 0.008 & 0.034 & 0.101 & 0.017 & 0.017 \\
\hline $\mathrm{Zn}$ & 0.084 & 0.006 & 0.018 & 0.008 & 0.083 \\
\hline $\mathrm{Se}$ & 0.003 & 0 & 0 & 0.002 & 0 \\
\hline $\mathrm{Br}$ & 0.058 & 0.015 & 0.021 & 0.012 & 0.057 \\
\hline $\mathrm{Sr}$ & 0.047 & 0.271 & 0 & 0.003 & 0 \\
\hline
\end{tabular}

Fuente: Autores. 
Tabla 7. Concentración de Minerales PRESEntes En MUestras DE PARTIDA Y BENEFICIADAS QUÍMICAMENTE PROVENIENTE DE C.

\begin{tabular}{|c|c|c|c|c|c|}
\hline Elemento & Partida & $\mathrm{C} 100$ & $\mathrm{C} 101$ & $\mathrm{C} 110$ & $\mathrm{C} 111$ \\
\hline $\mathrm{Al}$ & 7.433 & 0 & 0 & 0 & 0 \\
\hline $\mathrm{Si}$ & 17.733 & 0 & 0 & 0 & 0 \\
\hline $\mathrm{Cl}$ & 0 & 10.17 & 7.465 & 8.915 & 18.285 \\
\hline $\mathrm{K}$ & 0.601 & 0.183 & 0.463 & 0.114 & 0 \\
\hline $\mathrm{Ca}$ & 0.461 & 0.152 & 0.041 & 0.223 & 0.359 \\
\hline $\mathrm{Ti}$ & 0.718 & 0.617 & 0.181 & 0.417 & 0.683 \\
\hline $\mathrm{Cr}$ & 0.008 & 0 & 0.125 & 0.015 & 0.066 \\
\hline $\mathrm{Mn}$ & 0.010 & 0 & 0 & 0 & 0.034 \\
\hline $\mathrm{Fe}$ & 9.473 & 4.470 & 2.960 & 3.542 & 6.365 \\
\hline $\mathrm{Cu}$ & 0.006 & 0.025 & 0.015 & 0.035 & 0.040 \\
\hline $\mathrm{Zn}$ & 0.065 & 0.005 & 0.057 & 0.014 & 0.006 \\
\hline $\mathrm{Br}$ & 0.061 & 0.072 & 0.010 & 0.044 & 0.016 \\
\hline $\mathrm{Sr}$ & 0.037 & 0 & 0 & 0 & 0.002 \\
\hline
\end{tabular}

Fuente: Autores.

\section{CONCLUSIONES}

Para el beneficio químico de los carbones antracíticos se encontró que las mejores condiciones de desmineralización utilizando $\mathrm{HCl} 5 \mathrm{M}, \mathrm{HF} 40 \%$ y $\mathrm{HCl} 38 \%$ son 60 minutos como tiempo de reacción para cada ácido y un tamaño de partícula de $250 \mu \mathrm{m}$. Bajo estas condiciones se pueden alcanzar valores de $0.65 \%$ y $0.76 \%$ de cenizas (bs), para los carbones provenientes de minas de Boyacá y Santander (Colombia) respectivamente. La reducción del tamaño de partícula mejora los resultados finales de la remoción de materia mineral y cenizas. Se determinó que el tamaño de partícula ejerce una mayor influencia en la remoción de cenizas y materia mineral que el tiempo de reacción para cada ácido. El beneficio químico conduce a la reducción de compuestos de tipo silicatos, aluminatos y aluminosilicatos en un $100 \%$ y compuestos férricos por encima del 50\%.

Para futuras investigaciones se sugiere incluir el estudio de la influencia de diferentes ácidos y bases en la remoción de compuestos y materia mineral tales como ácido nítrico, ácido sulfúrico, hidróxido de sodio. Esto con el fin de identificar qué tipo de solución (ácido o base) puede emplearse para remover elementos específicos para la aplicación que se necesite. Esto puede utilizarse para procesos de grafitización y carbonización, en la industria metalúrgica y afines [8], [15], [16].

\section{FinANCIAMiento}

Artículo de Investigación derivado del proyecto titulado: "Efectos catalíticos de la materia mineral sobre la reactividad del carbón, biomasa y mezclas de carbónbiomasa en procesos de oxidación y devolatización térmica. Determinación de parámetros cinéticos mediante análisis termogravimétrico.» Financiado por la Universidad del Atlántico, Barranquilla, Colombia. Fecha Inicio: Agosto de 2016, Fecha Finalización: Diciembre de 2017.

\section{REFERENCIAS}

[1] J. M. Andrésen, C. E. Burgess, P. J. Pappano and H. H. Schobert, "New directions for non-fuel uses of anthracites," Fuel Processing Technology, vol. 85, no. 12, pp. 1373-1392, Aug. 2004. https://doi.org/10.1016/j.fuproc. 2003.05 .001

[2] W. Xia, G. Xie and Y. Peng, "Recent advances in beneficiation for low rank coals," Powder Technol., vol. 277, pp. 206-221, Jun. 2015. https://doi.org/10.1016/j.powtec.2015.03.003

[3] I. M. Mejia-Villarreal, "Producción de carbón ultralimpio por desmineralización física y química", M. S. thesis, Dept. Ing. Quim., Universidad del Valle, Cali, Colombia, 2004.

[4] M. Alfaro-Domínguez, F. J. Higes-Rolando, M. L. RojasCervantes and V. Gómez-Serrano, "Demineralisation of semi-anthracite char with molten salts/HCl. Effects on the porous texture and reactivity in air," Appl. Surf. Sci., vol. 252 , no. 17, pp. 6005-6008, Jun. 2006. https://doi. org/10.1016/j.apsusc.2005.11.002

[5] J. W. Leonard, Coal preparation. Society for Mining, Englewood, Colorado, USA: Metallurgy and Exploration, 1991.

[6] M. C. Vanegas Chamorro, "Estudio del mecanismo de grafitización de antracitas sudafricanas," M. S. thesis, Dept. Ing. Quim., Universidad de Oviedo, Oviedo, España, 2012

[7] P. Meshram, B. K. Purohit, M. K. Sinha, S. K. Sahu and B. D. Pandey, "Demineralization of low grade coal - A review," Renew. Sustain. Energy Rev., vol. 41, pp. 745-761, Jan. 2015. https://doi.org/10.1016/j.rser.2014.08.072

[8] S. K. Behera, S. Chakraborty and B. C. Meikap, "Chemical demineralization of high ash Indian coal by using alkali and acid solutions," Fuel, vol. 196, pp. 102-109, May. 2017. https://doi.org/10.1016/j.fuel.2017.01.088

[9] M. K. Saini, P. K. Srivastava and N. Choudhury, "Development of Moisture and Ash Based Correlation for the Estimation of Mineral Matter in High Ash Indian Coal," Int. J. Clean Coal Energy, vol. 4, no. 2, pp. 33-42, May. 2015. https://doi.org/10.4236/ijcce.2015.42004

[10] B. C. Smith, Infrared Spectral Interpretation: A Systematic Approach. Boca Raton, Florida, USA: CRC Press Taylor and Francis Group, 1998.

[11] A. M. Puziy, O. I. Poddubnaya, A. Martínez-Alonso, A. Castro-Muñiz, F. Suárez-García and J. M. D. Tascón, "Oxygen and phosphorus enriched carbons from lignocellulosic material," Carbon N. Y., vol. 45, no. 10, pp. 1941-1950, Sep. 2007. https://doi.org/10.1016/j.carbon.2007.06.014

[12] H. Machnikowska, A. Krztoń, and J. Machnikowski, "The characterization of coal macerals by diffuse reflectance infrared spectroscopy," Fuel, vol. 81, no. 2, pp. 245-252, Jan. 2002. https://doi.org/10.1016/S0016-2361(01)001259

[13] G. Socrates, Infrared and Raman characteristic group frequencies: tables and charts. Hoboken, Nueva Jersey, USA: John Wiley \& Sons, 2004.

[14] P. C. Painter, M. Starsinic, E. Squires and A. A. Davis, "Concerning the $1600 \mathrm{~cm}-1$ region in the i.r. spectrum of coal," Fuel, vol. 62, no. 6, pp. 742-744, Jun. 1983. https:// doi.org/10.1016/0016-2361(83)90317-4 
[15] S. Zhang, Z. Chen, X. Chen and X. Gong, "Effects of ash/ $\mathrm{K} 2 \mathrm{CO} 3 / \mathrm{Fe} 2 \mathrm{O} 3$ on ignition temperature and combustion rate of demineralized anthracite," J. of Fuel Chemistry and Technol., vol. 42, no. 2, pp. 166-174, Feb. 2014. https:// doi.org/10.1016/S1872-5813(14)60013-X

[16] X. Gong and S. Zhang, "Changes in char structure due to inorganic matters during anthracite pyrolysis," Journal of Analytical and Applied Pyrolysis, vol. 127, pp. 170-175, Sept. 2017. https://doi.org/10.1016/j.jaap.2017.08.011

[17] P. Meshram, B. K. Purohit, M. K. Sinha, S. K. Sahu and B. D. Pandey, "Demineralization of low grade coalA review," Renewable and Sustainable Energy Reviews, vol. 41, pp. 745-761, Jan. 2015. https://doi.org/10.1016/j. rser.2014.08.072
Wilmer Lugo Martinez recibió el título de Ingeniero Químico por la Universidad del Atlántico (Colombia). Sus intereses investigativos incluyen Beneficio y Proceso de transformación del carbón, Gestión eficiente de la energía, optimización de procesos y diseño de plantas industriales, simulación y modelamiento de procesos. Actualmente se desempeña como gestor energético en energía eficiente. https://orcid.org/00000002-7957-6090

Huber Ávila Rios recibió el título de Ingeniero Químico por la Universidad del Atlántico (Colombia). Sus intereses investigativos incluyen Beneficio y Proceso de transformación de carbón y Gestión eficiente de la energía. Actualmente se desempeña como de producción en Bimbo de Colombia. https://orcid.org/00000001-9039-1516

Marley Cecilia Vanegas Chamorro es Ingeniera Químico de la Universidad del Atlántico (Colombia). Magister en Ingeniería Química de la Universidad del Valle (Colombia) y Especialista en Gestión Eficiente de Energía con doctorado en Tecnología, Diversificación, calidad y Ahorro Energético por la Universidad de Oviedo (España). En la actualidad investiga en las áreas de Obtención de nuevos materiales a partir del carbón mineral, Eficiencia energética y fuentes no convencionales de energía. https://orcid.org/00000002-0513-7554

Alberto Albis Arrieta graduó en Ingeniería Química en la Universidad Nacional (Colombia) y como Doctor en Ciencias-Química de la misma universidad. Actualmente se desempeña como profesor Asociado en la Facultad de Ingeniería de la Universidad del Atlántico donde también lidera el grupo Bioprocesos. Sus intereses de investigación incluyen remoción de contaminantes y pirolisis. https://orcid.org/0000-0003-1758-1385

Marco Ardila Barragán es Ingeniero en Metalurgia (UPTC-Tunja - Colombia), Magister en Ingeniería Mecánica (UNINORTE-Barranquilla - Colombia). Profesor Escuela de Ingeniería Metalurgica de la UPTC. Investigador del Grupo de Investigación en Carbones y Carboquímica - Líneas de investigación: Pirólisis, Tecnologías limpias y Carboquímica. https://orcid. org/0000-0002-0251-7527 